\title{
Restocking Effects on the Single Species Population Obeying Difference Equation
}

\author{
Seiichi Watanabe* \\ (Accepted July 7, 1986)
}

\begin{abstract}
The restocking effects on a discrete growth population are considered in relation to two types (quadratic and Ricker) of reproduction system. Local stability analysis and numerical calculations are carried out to clarify the dynamics of the population. The stability depends on all parameters employed in the model. For the quadratic model, the restocking makes the equilibrium unstable, while for the Ricker model, the restocking produces stability. When increasing rate is high, and restocking level is low, then either population destruction occurs or crash cycles emerge. Restocking produces critical effects on population growth in some cases.
\end{abstract}

Recently, the sea farming has been widely practised in restocking of juveniles or youngs to natural populations. Theoretical studies are desired to clarify the restocking effects on the population fluctuation. There are a few investigations for restocking effects on predator-prey ${ }^{1)}$ and competition ${ }^{2-4)}$ systems. For the discrete population, the author investigated the restocking effects on the age structured abalone population. ${ }^{5 \text { ) }}$

Restocking the individuals to a natural population may produce various effects on the fluctuation of the population. Many species such as ice goby Leucopsarion petersi, pink salmon Oncorhynchus gorbusha, ayu fish Plecoglossus altivelis and so on, have non overlapping generation. Those species construct discrete system. We consider a simple discrete population model to clarify the dynamics of the system with restocking.

The simple discrete population without restocking is described as follows.

$$
X_{t+1}=f\left(X_{t}\right) \text {, }
$$

where, $X_{t}$ is number of individuals at time $t$ in reproduction system.

The quadratic and Ricker $^{\text {b) }}$ equations are widely used to describe the dynamics of the population. Those are as

$$
f\left(X_{t}\right)=A\left(1-B X_{t}\right) X_{t}
$$

and

$$
f\left(X_{t}\right)=A X_{t} e^{-B X_{t}} .
$$

Here, $A$ and $B$ are positive parameters. The dynamics of these equations are researched well. As parameter $A$ increases from small value to large one, the population behaves as stable point, stable cycles and chaos. ${ }^{7-11)}$

In this article, we consider the discrete population and clarify the restocking effects on the system.

\section{Model}

When we introduce restocking individuals to the population, the dynamics is described as

$$
X_{t+1}=f\left(X_{t}\right)+R \text {. }
$$

Here, the restocking level $R$ is assumed constant.

Clearly, constant restocking lifts the $f(X)$ curve upward by $R$ degree on the $\left(X_{t}, X_{t+1}\right)$ plane (Fig. 1).

For the quadratic equation, the slope of the $f(X)$ curve, $d f(X) / d X$, decreases linearly as $X$ increases. That is, from Eq. (2),

$$
\frac{d f\left(X_{t}\right)}{d X_{t}}=A\left(1-2 B X_{t}\right), \quad X_{t}>0 .
$$

The gradient is positive in the low population level $(X<1 / 2 B)$, zero at $X=1 / 2 B$, and negative in the high level $(X>1 / 2 B)$ (Fig. $2-I$ ).

For the Ricker equation, gradient of the curve is, from Eq. (3),

$$
\frac{d f\left(X_{t}\right)}{d X_{t}}=A\left(1-B X_{t}\right) e^{-B X_{t}}, \quad X_{t}>0 .
$$

The gradient is zero at $X=1 / B$ and smallest at $X=2 / B$ (Fig. 2-II).

\section{Equilibrium and Maximum Level}

The straight line in Fig. 1 is $X_{t+1}=X_{t}$. So, intersect point of this line and $f(X)$ curve is a

* Department of Aquatic Bioscience and Mariculture, Tokyo University of Fisheries, Konan, Minato, Tokyo 108, Japan (液㟫精一: 東京水産大学 凟源增殖学科). 


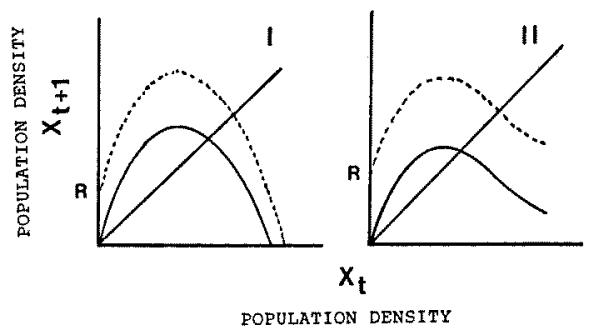

Fig. 1. Reproduction curve before (solid line) and after (dotted line) restocking.

I: quadratic model, II: Ricker model.

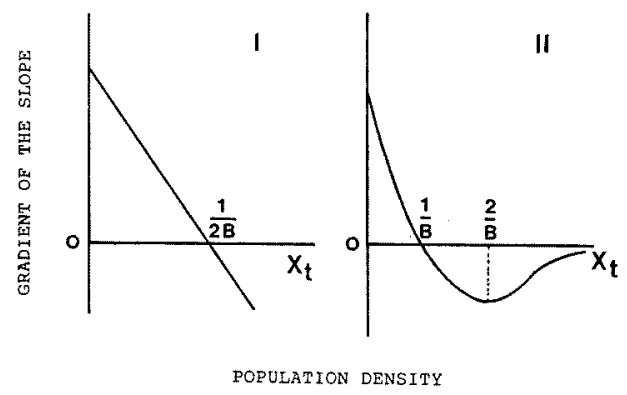

Fig. 2. Gradient of the slope of the reproduction curve.

I: quadratic model. II: Ricker model.

stationary point. That satisfies

$$
X^{*}=f\left(X^{*}\right)+R .
$$

For the quadratic equation, the equilibrium level is as

$$
X^{*}=\frac{A-1+\left\{(A-1)^{2}+4 A B R\right\}^{1 / 2}}{2 A B} .
$$

For Ricker equation equilibrium level is the value that satisfies following equation.

$$
X^{*}\left(1-A e^{-B X^{*}}\right)-R=0 .
$$

In both cases, $X^{*}$ increases as $R$ increases.

Maximum population level is as follows. For the quadratic equation, maximum population level, $X_{\max Q}$, is attained at $X=1 / 2 B$.

$$
X_{\text {rnaxQ }}=\frac{A}{4 B}+R .
$$

For the Ricker equation, population level is maximum when $X=1 / B$.

$$
X_{\max R}=A(B e)^{-1}+R \text {. }
$$

\section{Stability of the Equilibrium}

Local stability is defined by the behavior of

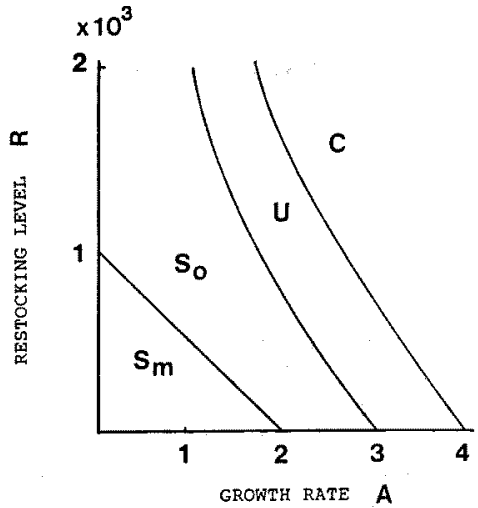

Fig. 3. Stability of the equilibrium for the quadratic model. $\mathrm{S}_{\mathrm{m}}$ : stable (convergent monotonically) $\mathrm{S}_{\mathrm{o}}$ : stable (convergent oscillatory) $\mathrm{U}$ : unstable $\mathrm{C}$ : crash.

small perturbed value from the equilibrium. Denoting $X_{t}=X^{*}+x_{t}$, and substituting this into Eq. (4) and neglecting 2nd and higher order terms of $x_{t}$, then the characteristic equation is obtained. For the quadratic equation,

$$
x_{t+1}-\left[1-\left\{(A-1)^{2}+4 A B R\right\}^{1 / 2}\right] x_{t}=0 .
$$

The characteristic root is

$$
\lambda_{Q}=1-\left\{(A-1)^{2}+4 A B R\right\}^{1 / 2} .
$$

So, the stability of the equilibrium is as follows.

(a) $0<\lambda_{Q}<1$, or $-1<A(A-2+4 B R)<0$. stable (convergent monotonically)

(b) $-1<\lambda_{Q}<0$, or $0<A(A-2+4 B R)<3$. stable (convergent oscillatory)

(c) $\lambda_{Q}<-1$, or $A(A-2+4 B R)>3$. unstable (divergent oscillatory)

The stability domain of the equilibrium for the quadratic model is as in Fig. 3.

For the Ricker model, characteristic equation is as

$$
x_{t+1}+\left(1-\frac{R}{X^{*}}\right)\left(B X^{*}-1\right) x_{t}=0 .
$$

Characteristic root is as

$$
\lambda_{R}=\left(1-B X^{*}\right)\left(1-\frac{R}{X^{*}}\right) .
$$

When $B$ is small value, the stability of the equilibrium is determined in a same manner as above case.

\section{Crash Cycles}

For the quadratic equation, the population 


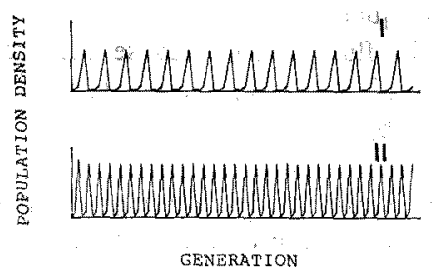

Fig. 4. Crash cycles of the population.

I: quadratic model, $A=5, R=10$. II: Ricker model, $A=60, X_{\mathrm{min}}=10, R=11$.

crashes when maximum population level exceeds the critical value $\hat{X}$ that satisfies $f(X)+R=0$.

$$
\hat{X}=\frac{1}{2 A B}\left\{A+\left(A^{2}+4 A B R\right)^{1 / 2}\right\} \text {. }
$$

So, the condition of crash is as

$$
X_{\max }>\hat{X} \text {. }
$$

This reduces to

$$
R^{2}-\frac{1}{2 B}\left(\frac{2}{A}-A+2\right) R+\frac{A}{16 B^{2}}(A-4)>0 .
$$

When $R=0$,

$$
A>4 \text {. }
$$

In this case, the critical level does not depend on the parameter $B$.

When the condition (18) is satisfied, the population crashes down, but it is unlikely that the population becomes negative. So, we assume here $X=0$ if $X<0$ under the condition of (18).

The constant restocking ensures the population to be positive, so under the condition of (18), the population repeats growth and crash (Fig. 4).

For the Ricker equation, the minimum population level, $X_{\text {m1n, }}$ is attained just next generation of $X_{\text {max }}$ if the population exhibit the cyclic fluctuation. $X_{\min }$ is as

$$
X_{\mathrm{man}}=A X_{\max } e^{-B X_{\max }} .
$$

When $X_{\min }$ is less than some value under which the population cannot persist, the population crashes to zero. The population repeats growth and crash (Fig. 4) when the restocking level slightly exceeds the $X_{\text {min }}$.

\section{Numerical Example}

Numerical calculations are carried out to clarify the dynamics of the system. For the quadratic equation, parameters $A$ and $B$ are fixed to the

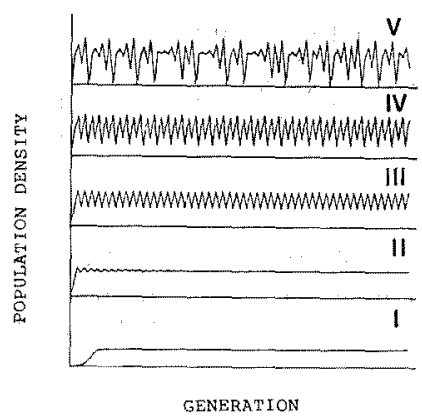

Fig. 5. Spectrum of the dynamical behavior of the population density as a function of time described by the quadratic equation for various values of parameter $R$, I: 0, II: 700, III: 1000 , IV: 1300 , $\mathrm{V}: 1618$.

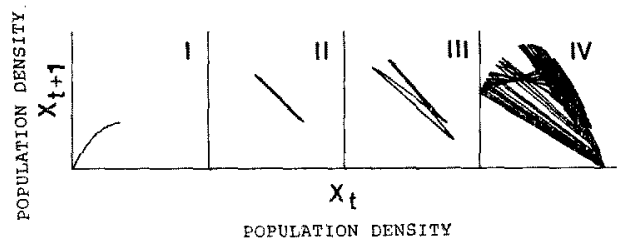

Fig. 6. Phase diagram of the population fluctuation described by the quadratic equation for various values of parameter $R$, I: 0 , II: 1000 , III: 1300 , IV: 1618 .

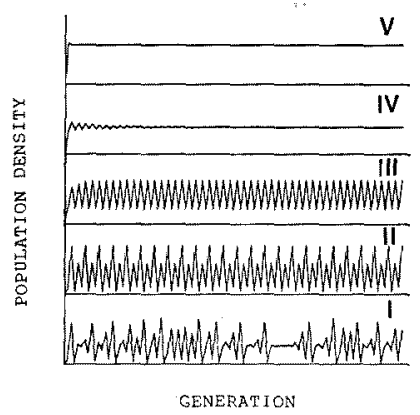

Fig. 7. Spectrum of the dynamical behavior of the population density as a function of time described by the Ricker model for various values of parameter $R$. I: 0 , II: 80 , III: 700 , IV: 1000 , V: 2000.

value of 2 and $5 \times 10^{-4}$ respectively. The initial population density is assumed to be 10 . While restocking level is low, the population settles to a stable equilibrium (Figs. 5-I, II and 6-I). $R$ increases, then periodical cycles occur (Figs. 5-III, IV and 6-II, III). Finally the population exhibits a chaotic behavior (Figs. 5-V and 6-IV). 


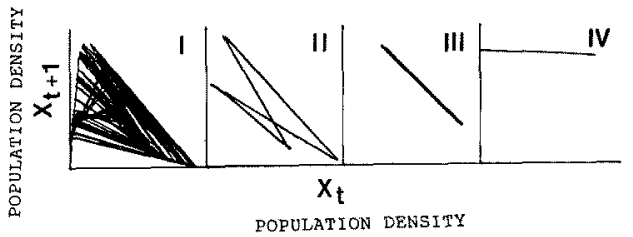

Fig. 8. Phase diagram of the population fluctuation described by the Ricker equation for various values of parameter $R$. I: 0 , II: 80 , III: 700, IV: 2000.

For Ricker equation, parameters $A$ and $B$ are fixed to 20 and $3 \times 10^{-3}$ respectively. If $R=0$, the population exhibits a chaotic behavior (Figs. 7-I and 8-I). When restocking is increased to some level, the periodical cycles occur (Figs. 7-II, III and 8-II, III). Finally, for sufficient restocking level, the population settles to a equilibrium (Figs. 7-IV, $V$ and 8-IV).

\section{Restocking Effects}

The restocking operation that introduce individuals to a population changes the stability of the population.

For the quadratic model, the fluctuation of the population is violated as restocking level increases. In this case, dynamics of the population is complicated by restocking operation.

While, in the case of the Ricker type renewal equation, the population is stabilized by restocking operation although there exists the case that violates the fluctuation of the population in low restocking level. Consider the population whose equilibrium is stable at $R=0$ level. When restocking operation is practised, then the gradient of the $f(X)$ curve at equilibrium point becomes steeper as $R$ increases at first, after then, $R$ increases much more, the gradient becomes gentle. So, for such population, as $R$ increases, the stable equilibrium is observed initially, then it is succeeded by oscillations, and finally settles to a stable equilibrium.

If accepting the assumption described in the crash cycle section, the population with high increasing rate, $A$, exhibits cyclic behavior of growth and crash.
For the quadratic model, restocking operation violates the fluctuation, because the gradient of the slope of the $f(X)$ curve at equilibrium is steepened by increasing $R$. So, growth and crash occur by turns.

For the Ricker model, although the population behaves same as the case of the quadratic equation while restocking is in low level, the fluctuation of the population is stabilized by intense restocking operation.

It is recognized that restocking effects on a population is different for these models. Therefore, the reproduction process must be investigated fully before restocking operation is practised. There is the case that the population is collapsed by harvesting. ${ }^{12)}$ Here, we also criticize that the restocking operation collapses the population in some case. So, careful restocking is desired.

\section{Acknowledgements}

I thank Prof. N. Hirayama (Tokyo University of Fisheries) for his helpful discussions and review of the manuscript.

\section{References}

1) F. Brauer and A. C. Soudack: I. Math. Biol., 11, 1-14 (1981).

2) S. Watanabe, R. Matsunaga, and F. Fushimi: $J$. Tokyo Univ. Fish., 68, 15-23 (1982).

3) S. Watanabe: J. Tokyo Univ. Fish., 70, 59-69 (1983).

4) S. Watanabe: J. Tokyo Univ. Fish, , 72, 57-63 (1985).

5) S. Watanabe: Res. Popul. Ecol. 28, 117-133 (1986).

6) W. E. Ricker: J. Fish. Res. Board Can., 11, 559-623 (1954).

7) R. M. May: Science, 186, 645-647 (1974).

8) R. M. May: J. Theor. Biol., 51, 511-524 (1975).

9) R. M. May and G. F. Oster: Amer. Nat., 110, 573-599 (1976).

10) J. Guckenheimer, G. Oster, and A. Ipaktchi: $J$. Math. Biol., 4, 101-147 (1977).

11) B. Bunow and G. H. Weiss: Math. Biosci., 47, 221-237 (1979).

12) R. M. Peterman: J. Fish. Res. Board Can., 34, 1130-1142 (1976). 